Case Report

\title{
Spontaneous and Simultaneous Bilateral Ruptured Tubal Ectopic Pregnancy: A Case Report
}

\author{
Zidane Farah", Cherkaoui Malki Nivine, Sebti Mamoun, Yousfi Mounia, Bargach Samir \\ Department of Obstetrics and Gynecology, Maternity Souissi Ibn Sina, University Mohammed V, Rabat, Morocco \\ Email address: \\ farahzidane@gmail.com (Z. Farah) \\ ${ }^{*}$ Corresponding author
}

\section{To cite this article:}

Zidane Farah, Cherkaoui Malki Nivine, Sebti Mamoun, Yousfi Mounia, Bargach Samir. Spontaneous and Simultaneous Bilateral Ruptured Tubal Ectopic Pregnancy: A Case Report. Journal of Gynecology and Obstetrics. Vol. 9, No. 1, 2021, pp. 1-4.

doi: $10.11648 /$ j.jgo.20210901.11

Received: October 1, 2020; Accepted: November 13, 2020; Published: January 15, 2021

\begin{abstract}
Simultaneous bilateral tubal ectopic pregnancy is a rare entity. The reported incidence ranges between 1 in 725 to 1 in 1580 of all ectopic pregnancies, and it is higher in women undergoing assisted reproductive techniques or ovulation induction. Its spontaneous appearance is almost exceptional. There are no unique features in clinical presentation helping to distinguish it from unilateral ectopic pregnancy. This makes the pre-operative diagnosis difficult, so it's commonly made during surgery. Whether the ectopic pregnancy is spontaneous or induced, fast management is essential and includes early diagnosis, detailed ultrasound scan, careful contralateral tube control, and also specimen's histological verification. Thus the treatment will depend on the hemodynamic stability of the patient, the extent of tube damage, as well as the desire for future pregnancies. We report a case of 40 year old patient, gravida 1 without any ectopic pregnancy risk factors, who suffered from abdominal pain and vaginal bleeding at 8 gestation weeks. Endovaginal ultrasound detected adnexal ruptured mass in the right fallopian tube. Urgent laparotomy was performed for suspicious ruptured ectopic pregnancy. A right and left ruptured ectopic pregnancy were found during operation. Bilateral salpingectomy is done and patient is discharged from hospital 3 days later without complications.
\end{abstract}

Keywords: Ectopic Pregnancy, Ruptured Fallopian Tube, Bilateral Salpingectomy

\section{Introduction}

Bilateral ectopic pregnancy is a rare condition in which there are at least 2 concomitant pregnancies in the same patient, with both being located in opposite uterine appendages. The first case of primary bilateral ectopic pregnancy described was published by Bledsoe in 1918 [1]. The bilateral tubal ectopic pregnancy reported instance is 1 in 200000 pregnancies, and more than half of those cases were the result of assisted reproductive technology (ART). However, the spontaneous form is exceptional and the first case was described in Colombia in 1997 [2]. The clinical presentation is unpredictable and its detection is urgent because of the associated morbidity and mortality due to the rupture. We report a case of spontaneous bilateral ruptured tubal ectopic pregnancy which was diagnosed during surgery.

\section{Case Report}

A 40 year-old woman, gravida 1, was admitted to the gynecological emergency with a history of 8 weeks amenorrhea, intermittent vaginal bleeding and mild lower abdominal pain of 10 days duration. She had been married for 8 years. She had no history of sexually transmitted infection and no irregularities in menstruation; she also denied having received infertility treatment at this moment. There was no contraception use or previous abdomino-pelvic surgery.

General examination revealed maternal pallor with tachycardia: pulse 108 per minute, the blood pressure was normal and her abdomen was tender on palpation. The pelvic examination showed a long posterior closed cervix with mild spotting, a normal-sized uterus, but the adnexa were difficult to palpate. Initial serum BHCG was $11868 \mathrm{UI} / \mathrm{ml}$ and 
haemoglobin 9g/dl. Transvaginal ultrasound found no evidence of intrauterine pregnancy, a tick endometrium of $10 \mathrm{~mm}$ (Figure 1), and a right side adnexal mass with a diameter of $37 / 46 \mathrm{~mm}$ (Figure 2). A moderate amount of fluid collection was present in the pouch of Douglas.

Clinical and radiological findings were suggestive of ectopic tubal pregnancy. Emergency exploratory laparotomy revealed haemoperitoneum of approximately $800 \mathrm{ml}$ with bilateral ectopic pregnancy. The right fallopian tube was found to contain a mass in the ampullary region measuring $4 / 3 \mathrm{~cm}$ ruptured on the upper side. The left fallopian tube also showed a ruptured fimbrial ectopic pregnancy that was bleeding and forming an organized haematoma (Figure 3).

In view of these findings a bilateral salpingectomy was carried out (Figures 4 and 5). The patient received two concentrates of red blood cells. Postoperative follow up was uneventful and the patient was discharged on the $5^{\text {th }}$ day. Three weeks after surgery the BHCG was zero.

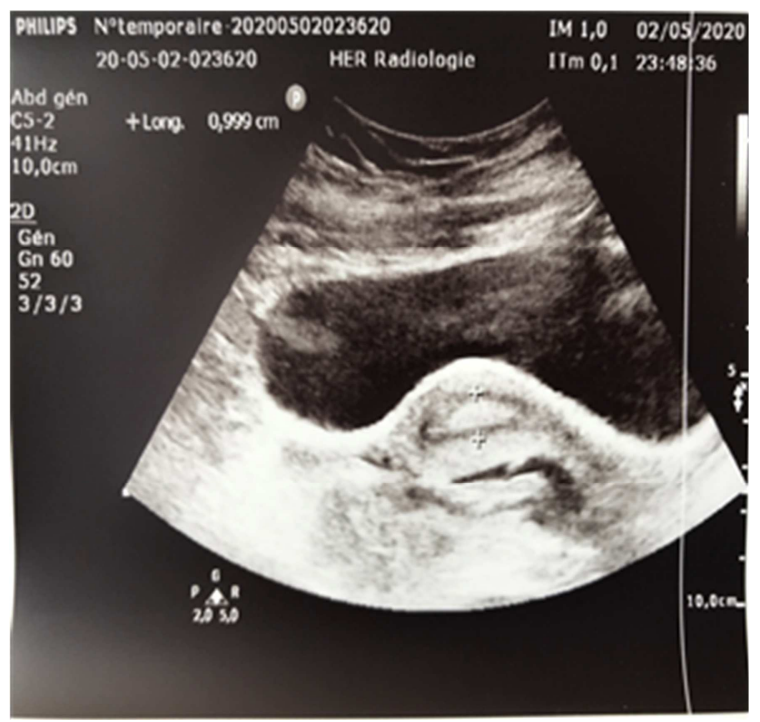

Figure 1. Thick endometrium of $10 \mathrm{~mm}$.

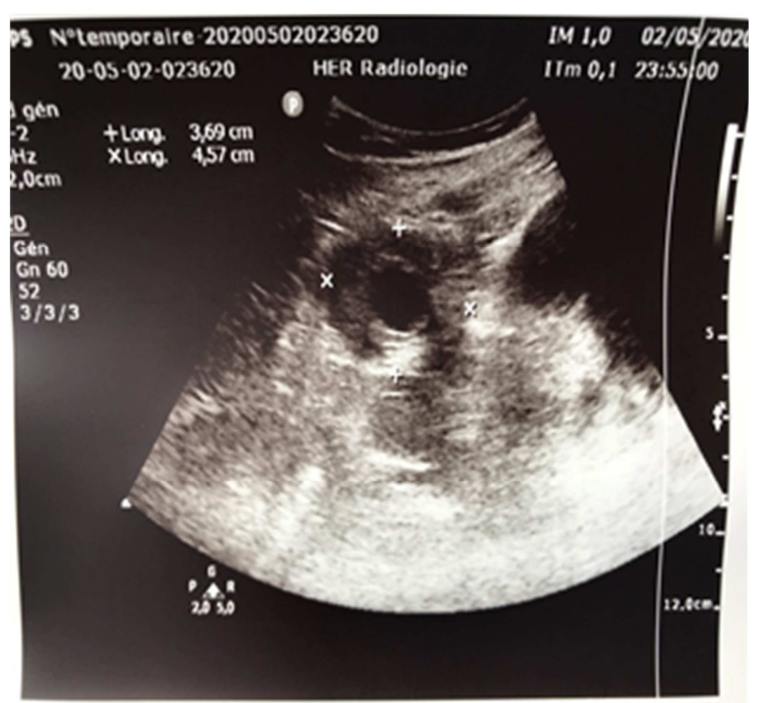

Figure 2. Right side adnexal mass.

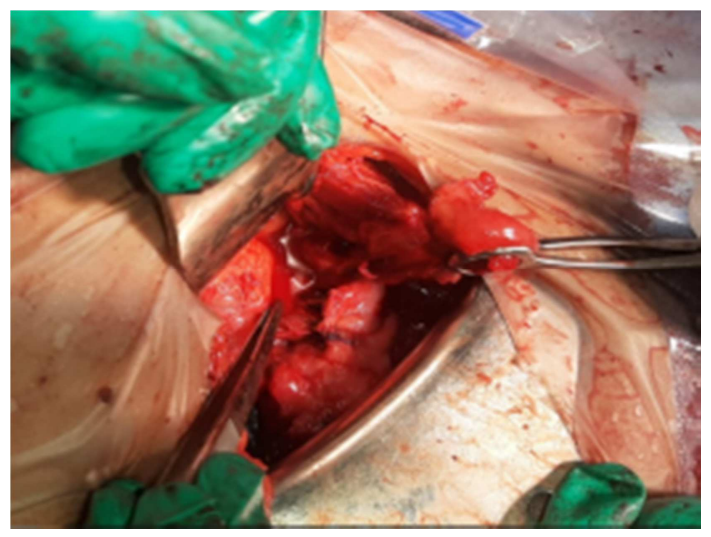

Figure 3. Ruptured fimrial ectopic pregnancy.

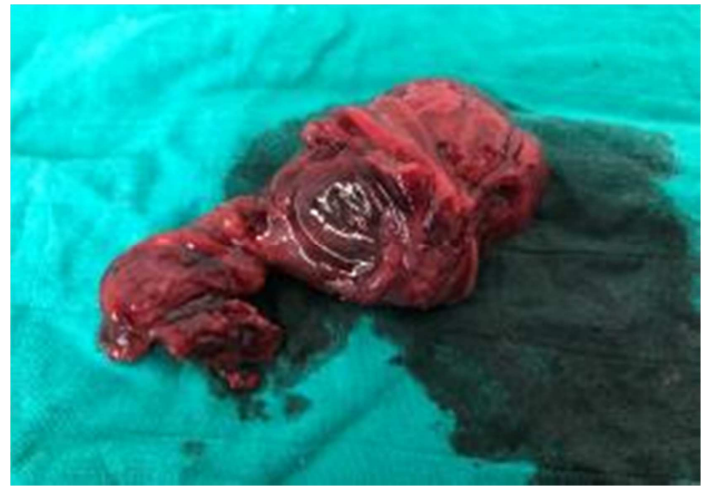

Figure 4. Right fallopian tube.



Figure 5. Left fallopian tube.

\section{Discussion}

The frequency of ectopic pregnancy has increased considerably since the $70 \mathrm{~s}$; it represents $2 \%$ of all pregnancies. The bilateral ectopic pregnancy incidence ranges from $1 / 725$ to $1 / 1,580$; this rate increased in the last decades and the main risk factor seems to be the use of reproductive techniques. However José's 2007 review shows that in the last 26 years, $43 \%$ of the reported bilateral ectopic pregnancies have been spontaneous [1]. The preoperative diagnosis is uncommon and late treatment leads to maternal death; so whether the ectopic pregnancy is spontaneous or induced, good management includes early presentation, a high index of suspicion, detailed ultrasound scan, careful intraoperative inspection, histological verification of specimens, and appropriate patient counseling 
[3].

Several etiology theories have been postulated regarding bilateral tubal ectopic pregnancy, as the transperitoneal migration of trophoblastic cells from one tube to another [4], multiple ovulation reported by Foster et al. [5], and superfetation which is the development of a second oocyte in an already pregnant woman [6]. Our patient has none known risk factor of ectopic pregnancy, which is the case of approximately half of all women with the same condition as reported by Barnhart KT's study in 2006 [9]. The diagnostic criteria of this entity were first defined by Fishback in 1939 [7] who proposed that 2 embryos should be observed in any portion of the tubes, accompanied by chorionic tissue. Fourteen years later Norris argued that the aforementioned diagnosis could be reached just by finding chorionic villi in surgical specimens from both tubes [8].

It is difficult to differentiate a bilateral from unilateral tubal ectopic pregnancy before surgery. Neither clinical symptoms, blood levels of BHCG or even ultrasonography are specific of the bilateral condition [10]. These three entities in our case failed to make such a diagnosis and this is in agreement with other reports. In a reported case review of 18 patients, 9 were diagnosed from a lack of correlation between ultrasound findings and B-hCG levels and were symptom free or presented minor clinical signs. Only half cases arrived with a clinical picture of acute abdomen or hypovolemic shock [1]. Also, it is possible to establish a correlation between BhCG values and the gestational age measured in weeks, beginning from the date of the last menstruation, but the ample range of BHCG concentration makes it difficult to recognize a bilateral ectopic pregnancy only on the basis of its value. In Marpeau's study, only $1(12.5 \%)$ of the 9 values found and correlated with gestational age was abnormally high [11]. Neither can the use of ultrasound be advocated as standard of care in the diagnosis of bilateral ectopic pregnancy. It could detect unilateral ectopic pregnancy in about $90 \%$ of cases. One study reported that transvaginal sonography showed adnexal abnormality in nearly $95 \%$ of cases with ectopic pregnancies; the most common finding ( $>50 \%)$ was nonspecific adnexal mass [12]. Therefore, diagnosis of bilateral ectopic pregnancy continues to be an important challenge facing emergency physicians.

Since ultrasound and levels of beta fraction of gonadotropin-chorionic hormone (B-HGC) do not provide enough information to differentiate a unilateral from a bilateral ectopic pregnancy, laparoscopy if possible or laparotomy is usually the most frequent method for diagnosis bilateral tubal pregnancy. So a careful exploration of both tubes should be performed during surgery. The treatment will depend on the hemodynamic stability of the patient, the extent of tube damage, as well as the desire for future pregnancies. In case of hemodynamic instability, emergency surgery must be performed; on the other hand, if the patient is stable, a diagnostic approach is necessary in order to choose a surgical, a conservative or an expectant management.

Expectant method consists of monitoring the BHCG blood level at first every two days then weekly until recovery (until the hCG level falls below $2 \mathrm{IU} / \mathrm{L}$ ). This method is selected if there is no gestational sac at transvaginal ultrasound and if BhCG level is less than $200 \mathrm{mIU} / \mathrm{mL}$ [13]. Medical treatment with methotrexate (MTX) was also frequently described but reserved for special cases, several regimens are used: intramuscular injections of $50 \mathrm{mg} / \mathrm{m}^{2}$ in single or multiple doses based on different protocols as reported by Denise Harari in a recent study [13]. Another study described the use of combined ultrasound-guided local injection of MTX and systemic MTX as an efficient non-surgical option for women with tubal pregnancy, high serum $\beta$-hCG concentrations, and fetal cardiac activity [14]. Krissi H, also showed in her report the efficiency of combining uterine artery MTX infusion and embolization with systemic MTX for non-tubal ectopic pregnancies treatment in women who were still trying to conceive [15].

Nevertheless, surgical intervention is usually required and ranges from salpingectomy for one tube and linear salpingostomy for the other, to bilateral salpingostomy or bilateral salpingectomy, depending on the fallopian tube injury degree [16]. A study discussed the use of salpingectomy in cases with rupture, as was done for our patient and bilateral conservative surgery in unruptured ectopic tubal pregnancy. Salpingostomy is still preferred even if a multicenter, international, randomized controlled trial on women aged 18 years and above showed that it doesn't significantly improve fertility prospects compared with salpingectomy [17]. Careful attention should be directed to follow-up tests. A serial measurement of serum concentrations of human chorionic gonadotrophin is necessary to rule out the risk of persistent trophoblast. If postoperative concentration of human chorionic gonadotrophin is diagnosed, follow-up until complete resolution is necessary. Further medical treatment with methotrexate or surgery in symptomatic patients may be necessary if human chorionic gonadotrophin levels do not decline or persist [10]. The appropriate surgical procedure in a bilateral ectopic pregnancy must be considered, and the choice of fertility-preserving surgery must be weighed against the increased risk of recurrence which ranges from 6 to $16 \%$ [18].

\section{Conclusion}

Ectopic pregnancy continues to be an important health problem in obstetrics; it more significantly affects developing countries and has an impact on maternal morbidity and mortality. Although the incidence has been increasing since the advent of assisted reproduction techniques, it is important to notice that this rare and serious condition can also occur in patients with no risk factors and who never used ART. Mortality in developed countries has decreased due to laparoscopic surgery that allows timely diagnosis and fast treatment. Further studies and investigations are required to justify the best therapeutic choice and to prove whether unilateral or bilateral conservative fertility-preserving surgery is more appropriate. 


\section{References}

[1] José F. De Los Ríos, MD, Juan D. Castañeda, MD, and Aguirre Miryam, MD Bilateral ectopic pregnancy.

[2] De Los Ríos JF (2006), Castañeda JD, Restrepo E. Lineal laparoscopic salpingostomy for treating spontaneous bilateral ectopic pregnancy: a case report. Rev Colomb Obstet Ginecol.

[3] Eze J N (2012), Obuna J A, Ejikeme B N. Bilateral tubal ectopic pregnancies: a report of two cases. Ann Afr Med.

[4] Tabachnikoff R M, Dada M O, Woods R J. et al. Bilateral tubal pregnancy. A report of an unusual case. J Reprod Med.

[5] Foster H M (1982), Lakshin A S, Taylor W F. Bilateral tubal pregnancy with vaginal delivery. Obstet Gynecol.

[6] Baijal N (2007), Sahni M, Verma N. et al. Discordant twins with the smaller baby appropriate for gestational age-unusual manifestation of superfetation: a case report. BMC Pediatre.

[7] Fishback HR (1939). Bilateral simultaneous tubal pregnancy. Am J Obstet Gynecol.

[8] Norris S (1953). Bilateral simultaneous tubal pregnancy. Can Med Assoc J.

[9] Barnhart K T (2006), Sammel M D, Gracia C R. et al. Risk factors for ectopic pregnancy in women with symptomatic first-trimester pregnancies. Fertil Steril.

[10] G. A. Al-Quraan (2007), M. I. Al6Taani, B. M. Nusair, A. El-Masri, M. R. Arafat and M. M. Khateeb Spontaneous ruptured and intact bilateral tubal ectopic pregnancy.

[11] Marpeau O (2005), Barranger E, Cortez A, Uzan S. Bilateral tubal pregnancy after natural conception: a case report. J Reprod Med.

[12] Frates M C (2014), Doubilet P M, Peters H E. et al. Adnexal sonographic findings in ectopic pregnancy and their correlation with tubal rupture and human chorionic gonadotropin levels. J Ultrasound Med.

[13] Denise Niza (2017) Benardete Harari, Diego Meraz Ávila, Rolando Álvarez Valero, Fátima Rubio Tijerina Embarazo ectópico tubárico bilateral espontáneo.

[14] Wang M (2014), Chen B, Wang J. et al. Nonsurgical management of live tubal ectopic pregnancy by ultrasound-guided local injection and systemic methotrexate. J Minim Invasive Gynecol.

[15] Krissi H (2014), Hiersch L, Stolovitch N. et al. Outcome, complications and future fertility in women treated with uterine artery embolization and methotrexate for non-tubal ectopic pregnancy. Eur J Obstet Gynecol Reprod Biol.

[16] Saubhagya Kumar Jena (2016), Sweta Singh, Monalisha Nayak, Leena Das, and Swagatika Senapati Pregnancy: A Case Report, Review of Literature and a Proposed Management Algorithm.

[17] Mol F (2014), van Mello N M, Strandell A. European Surgery in Ectopic Pregnancy (ESEP) study group. Salpingotomy versus salpingectomy in women with tubal pregnancy (ESEP study): an open-label, multicentre, randomised controlled trial. Lancet.

[18] S. Hoffmann (2016), H. Abele, and C. Bachmann Spontaneous Bilateral Tubal Ectopic Pregnancy: Incidental Finding during Laparoscopy- Brief report and Review of Literature. 